\title{
LA EUTANASIA ENTRE EL ACORTAMIENTO DE LA VIDA Y EL APOYO A MORIR: EXPERIENCIAS DEL PASADO, RETOS DEL PRESENTE
}

\author{
Dietrich von Engelhardt
}

\begin{abstract}
Resumen: La eutanasia hace referencia a la autoimagen y a la imagen del mundo del hombre; también a la comprensión de la enfermedad y la muerte. En la antigüedad significaba una muerte honrosa y agradable y no el término de la vida activa. El arte de la muerte en el medioevo cristiano pertenece al arte de la vida. Y el que comprende la vida, también debe conocer la muerte. Estudios médicos, psicológicos y sociológicos han llevado, en la actualidad, a nuevos conocimientos sobre el trato con la muerte y el fallecimiento.

La muerte se refiere a diferentes ámbitos: desintegración física, aislamiento social, tristeza de vivir y miedo a la muerte. La muerte social puede ocurrir antes de la muerte física y la muerte espiritual antes de la muerte del alma.
\end{abstract}

Palabras clave: Eutanasia, vida activa, muerte, desintegración, aislamiento

\section{EUTHANASIA IN BETWEEN SHORTENING LIFE AND AIDING DEATH: PAST EXPERIENCES, PRESENT CHALLENGES}

\begin{abstract}
Euthanasia refers to man's self image and his world's image; also to understanding sickness and death. In ancient times it meant an honorable and pleasant death and not the end of an active life. The art of death in the Christian Middle Ages was part of the art of life. And he, who understands life, must know death too. Nowadays, medical, psychological and sociological studies have lead to new knowledge about handling death and dying. Death refers to different scopes: physical desintegration, social isolation, sadness of living and fear of death. Social death may happen before physical death and spiritual death before the soul's death.
\end{abstract}

Keywords: Euthanasia, active life, death, desintegration, isolation

\section{A EUTANÁSIA ENTRE O ABREVIAMENTO DA VIDA E O APOIO AO MORRER: EXPERIÊNCIA DO PASSADO, DESAFIOS PARA O PRESENTE}

Resumo: A eutanásia faz referência a autoimagem e a imagem de mundo que tem o homem; também refere-se a compreensão sobre a doença e a morte. Na antiguidade significou morte honrosa e agradável e não fim da vida ativa. A arte de morrer no medievalismo cristão pertence a arte da vida. Aquele que compreende a vida, também, conhece a morte. Atualmente, estudos médicos, psicológicos e sociológicos têm proporcionado novos conhecimentos sobre como lidar com a morte e o morrer.

A morte é representada em diferentes âmbitos: desintegração física, isolamento social, tristeza de viver e o medo da morte. A morte social pode ocorrer antes da morte física e a morte espiritual antes da morte da alma.

Palavras chave: Eutanásia, vida ativa, morte, desintegração, isolamento

\footnotetext{
* Director Institut fur Medizin-und Wissenschaftsgeschichte, Medizinische Universität zu Lübeck, Deutschland. Correspondencia: v.e@imwg.mu-luebeck.de
} 


\section{Contexto y Actualidad}

Hoy en día, se discute sobre la eutanasia en todo el mundo. La reglamentación legal de la eutanasia activa en los Países Bajos ha llevado a impetuosos debates. La eutanasia alude profundamente a la autoimagen y a la imagen del mundo del hombre, a la comprensión de la enfermedad y la muerte. Trata sobre la libertad y la subordinación, la naturaleza, la sociedad y la cultura.

El desarrollo histórico en las valoraciones y usos de la eutanasia ha sido diverso. Como opresiva e intimidante para el futuro permanece la experiencia del trato criminal llevado a cabo mediante la eutanasia durante la época del Tercer Reich, que significó finalmente la matanza de enfermos, lisiados y moribundos.

La eutanasia representa un gran reto para la humanidad, para la medicina de la sociedad del presente, para el médico, el personal auxiliar y los enfermos, un reto para todos los hombres y sus iguales debido al progreso científico, tanto técnico como natural, y el cambio demográfico con el aumento de adultos mayores e impedidos y la explosión de costos de la medicina y del sistema de salud pública.

Nuevas iniciativas y nuevas leyes manifiestan la gran actualidad de este tema. La población del Estado de Washington rechazó en los Estados Unidos, hace pocos años, con sólo un $55 \%$ contra un $45 \%$, la legalización de la ayuda a morir activa proporcionada por los médicos para el caso de pacientes desahuciados sin ninguna posibilidad de curación. En los Países Bajos, se ha abierto una posibilidad en el sentido del "consentimiento". Se aplaza el procesamiento penal de la eutanasia activa bajo determinadas circunstancias $\mathrm{y}$, al mismo tiempo, se concreta la penalidad de esta intervención, que es sustentada por la legislación de la reglamentación estatal de emergencia -en el sentido legal holandés-. El filósofo australiano Peter Singer llamó la atención con su libro Praktischen Ethik (1984) y provocó intranquilidad en los discapacitados cuando señaló que los fetos y los recién nacidos podrían ser vistos como menos valiosos, como "la vida de un cerdo, de un perro o de un chimpancé"(1) y justificó también la eutanasia activa en determinadas enfermedades mentales.

La comprensión y clasificación de la eutanasia no son controversiales sólo en nuestros días, también lo fueron en el pasado. La historia de las palabras nos permite reconocer cómo se hablaba de manera distinta de la eutanasia y ante todo: cuán positivo ha sido su sentido(2-4).

La muerte ha estado presente desde la antigüedad hasta el siglo XX en el círculo de la familia, amigos y vecinos. La muerte constituía un suceso social, una forma externa y una interpretación espiritual, pero la perspectiva religiosa mostraba a los agónicos como supervivientes que requerían de apoyo y consuelo. La interpretación no excluyó ni el miedo ni el dolor; quien aprueba la muerte puede sentirse atemorizado por el enfermo terminal, quien le encuentra un sentido al fallecimiento puede sentir un profundo dolor por la pérdida de un pariente o un amigo. El espectro de reacciones en torno a la muerte y el fallecimiento era en el pasado bastante considerable y, en nuestros días, es aún muy extenso $(5,6)$.

Las suposiciones políticas, científicas, legales y las causas culturales corresponden a influencias particulares. Siempre es necesario interrogarse sobre las nuevas influencias, qué valores espirituales y normas hacen imposible 
que la eutanasia sea justificable, no sólo teóricamente sino también como una práctica que a futuro podría hacerse presente. La mirada hacia la filosofía, posiciones teológicas y literarias e informes del pasado, así como también hacia causas económicas, políticas, sociales y psicológicas, aparece surgiendo de la preocupación por el presente y el futuro.

\section{Antigüedad}

La eutanasia surge como hecho y palabra ya en la antigüedad. Con la expresión griega "Euthanasia", el emperador romano Augusto, según Suetonio, designa su ideal de muerte, que él, a la edad de 76 años, también ha experimentado. " $¡$ Livia, recuerda nuestra feliz unión y vive feliz!"(7) deben haber sido las últimas palabras que se conocen del emperador. Tanto en poetas como filósofos de la antigüedad se encuentra esta expresión como adjetivo o substantivo.

Desde la antigüedad hasta nuestros días, parte esencial de la ética médica en relación con el nacimiento y la muerte es el Juramento de Hipócrates del siglo V/IV antes de Cristo. Eje central de este juramento, con sus prohibiciones y mandatos, es la negación de la eutanasia activa y la ayuda a cometer suicidio: " no le daré a nadie un remedio que pudiera causar la muerte, aunque se me pida, ni tampoco daré un consejo en esa dirección”.(8) El médico, no sólo debe preservar y proteger la vida, sino también evitar ponerla en peligro o acabar con ella. La promesa de los médicos de Ginebra de 1948 se inserta en esta tradición: "Respetaré la vida del hombre desde la concepción, incondicionalmente".

Bajo ningún punto de vista el Juramento de Hipócrates es obligatorio para todos los médicos. Incluso en la actualidad no corresponde, en momentos esenciales, a las convicciones de muchos hombres, médicos y trabajadores sanitarios. Ya en la antigüedad se sustentaban, además, otras posiciones. En el libro Politeia de Platón (375 a C.) se hablaba, categóricamente, aun cuando sin el uso de este concepto, de eutanasia activa y pasiva: "Implantarás tal jurisprudencia en la ciudad como una medicina, según hemos descrito, con el propósito de cuidar a los ciudadanos sanos de cuerpo y alma, pero que ya no lo están; sólo permite que la muerte ocurra cuando su alma ya se ha vuelto incurable e insana" (Politeia $409-410)$.

Según el sentido filosófico del Stoa, el médico tiene permiso para ayudar a morir de manera activa cuando el sufrimiento físico o mental amenazan el saber racional y las cuestiones morales. Famosos ejemplos de la antigüedad son los de los médicos que apoyaron el suicidio de Cato el joven y de Séneca. El ideal de la filosofía estoica es la aceptación de la muerte mientras haya vida ("mori discere"), que también sirve para los escritos antiguos sobre el trato hacia la muerte y el fallecimiento. En la antigüedad, eutanasia significaba, en esta perspectiva, una muerte honrosa y agradable ("felici vel honesta morte mori"), no así término de la vida activa.

\section{Edad Media}

El medioevo cristiano trajo consigo cambios de gran alcance para el trato con la muerte y el fallecimiento. Una profundidad, hasta ahora desconocida, de la piedad y del amor al prójimo - "misericordia" y "caritas" se introduce con el principio de la trascendencia en el mundo, así como también en la medicina. La eutanasia, el suicidio y el aborto son considerados como pecado, puesto que el hombre no puede disponer libremente sobre la 
vida, que le fue dada por Dios: "Yo soy el que da la vida y la quita" [32, 39]. La expresión eutanasia no se encuentra ni en el Antiguo ni en el Nuevo Testamento, tampoco se presenta en los escritos teológicos del medioevo. Cristo se transforma, con su desesperación y esperanza camino a su muerte, en una figura que da fuerza y consuelo a todos los que sufren y mueren; tampoco ha quedado libre del sufrimiento y de la muerte a causa del acortamiento de la vida.

El arte de la muerte ("ars moriendi"), en el medioevo cristiano, pertenece al arte de la vida ("ars vivendi"); el que comprende la vida, también debe conocer la muerte. La muerte repentina o imprevista ("mors repentina et improvisa"), deseo de muchos hombres de hoy, se considera como una muerte mala y horrenda ("mala mors"). Se ruega a Dios protección ante esta muerte: "Ab improvisa morte libera nos, Domine". Se desea estar consciente para despedirse de familiares y amigos, "con mano tibia" dar al heredero, y poder presentarse en el más allá con un claro conocimiento del fin de la vida.

La enfermedad y la muerte se aplican a la expresión "Passio Christi", en el proceso escatológico del paraíso sobre la vida terrenal deben unirse con la resurrección. La enfermedad puede tener un sentido positivo: a través de ella el hombre puede perfeccionarse en su virtud ("virtus in infirmitate perficitur", 2. 12, 9). En el medio de la vida estamos siempre ante la muerte ("Media vita in morte somus"), los vivos de hoy son los muertos de mañana. El arte de la muerte tiene algo de valentía, pero también de consuelo - pedido a los sacerdotes, parientes y médicos.

Entre las obras de misericordia cuenta la ayuda en la muerte y el entierro del fallecido. Las siete virtudes clásicas y cristianas
- sabiduría, valentía, humildad, equidad, fe, amor y esperanza - deben ser una ayuda en el trato con la muerte; sobre todo se debe destacar la esperanza que brinda el médico - en sentido tanto inminente como trascendente -; vivir significar tener esperanza, tener esperanza significa vivir ("spiro dum spero").

\section{Modernidad}

Los hombres de la Edad Moderna ya no siguen, como es sabido, el pensamiento de la Edad Media, en ocasiones sólo a un alcance parcial o mínimo. La perspectiva cristiana se abandona y se vuelve a posiciones filosóficas de la Antigüedad, quedando en primer lugar este mundo, la naturaleza y el hombre. La salud, la juventud y la vida eterna deben ser objetivos alcanzables con la ayuda de la técnica, de las ciencias naturales y de la medicina; de estas metas se alimenta la poderosa e ilimitada dinámica del progreso médico y científico natural. La muerte y el fallecimiento se convierten, crecientemente, en un suceso privado y perteneciente a este lado, cada vez más también se vuelve un acontecimiento silencioso y solitario, no siempre en un hecho simplemente biológico y objetivo.

De los grandes utopistas del Renacimiento vuelve a justificarse el término activo de la vida, condenado durante la Edad Media, y se relaciona con la expresión eutanasia. Los esbozos de Tomás Moro y Francis Bacon están, al mismo tiempo, llenos de sugerencias eugenésicas, tal como ya aparecía en el concepto de Estado de Platón. La eutanasia cambia, de ahora en adelante, a una piedra de tope fundamental de la imagen del hombre, del concepto de vida y de la comprensión de la ciencia, de la relación médico - paciente así como también de la solidaridad entre los hombres. Este hecho no 
ha experimentado cambios hasta nuestros días.

Bacon, que también usa la expresión "suicidio", toma en su escrito De dignitate et augmentis scientiarum del año 1623, por primera vez, la antigua designación de eutanasia, y diferencia dos tipos: la "eutanasia exterior" como término directo de la vida ("excessus e vita lenis e placidus"), y la "eutanasia interior" como preparación espiritual para la muerte ("animae praeparatio")(9). Con esto, Bacon se refiere, por una parte, a la tradición de "ars morendi", que siempre cuenta como un próspero "ars vivendi”, pero completa esta tradición con algo que para el medioevo era una posibilidad inimaginable: la muerte de un enfermo por manos del médico. Con el énfasis en la autonomía, Bacon indica el peligro de la heteronomía involuntaria, que él rechazara terminantemente.

Moro, quien también justifica el suicidio, ya en 1516, en su Utopía, aboga por la eutanasia activa, sin usar esta designación. La espontaneidad es un requisito decisivo de la eutanasia activa para Moro y Bacon; contra la voluntad del enfermo o sin aclaración, la eutanasia no puede tener lugar: "Quien se ha convencido de esto, quien termina su vida, ya sea voluntariamente a través de la abstención de recibir alimentos o es puesto a dormir y encuentra salvación sin darse cuenta de la muerte. Contra su voluntad no se debe matar a nadie, se le debe prestar cuidados igual que a cualquier otro"(10).

Sin embargo, no se encuentra ausente la contraparte de advertencia. De ninguna manera las utopías son, en su mundo y en la imagen del hombre, idénticas. En el siglo XVII, se sostiene una postura diferente a la de Platón, Moro y Bacon, la del teólogo Johann Valentin
Andreae; los habitantes de su utópico Cristianopolis, del año 1619, asignan el convencimiento religioso para los enfermos del cuerpo y del alma y no los rechazan de la colectividad humana: "Los hombres, cuyo espíritu está desorientado o alterado, sufren, cuando es tolerable; éste no es el caso, así lo logran bajo una suave vigilancia. Del mismo modo, esto se presenta con los deformes, que piden una razón del por qué la sociedad del hombre los trata con negligencia y no buenamente. Dios no nos hace sufrir con interminables bienes y paciencia, como El nos quiere, sino como nosotros somos"(11). Estas frases no han perdido su valor e, incluso en nuestros días, deben volver a ser recordadas.

Como en los próximos siglos los médicos del Renacimiento no siguieron a Moro ni a Bacon, rechazaron la eutanasia externa; justificaron la eutanasia pasiva; sobre todo exigieron la eutanasia interior. En el espectro de esta variedad de significados, la eutanasia se mueve siempre entre los extremos de un término físico y de una ayuda mental espiritual. A comienzos del siglo XIX, el médico Christoph Wilhelm Hufeland determina présbita y categóricamente: "El médico sólo debe preservar la vida, se trate de una suerte o de una desgracia, valga o no la pena. Esto no le concierne y, una vez más, se adjudica esta consideración en su labor de asimilar, así las consecuencias son enormes y el médico se transformará en el hombre más peligroso de la ciudad"(12).

La interpretación de la eutanasia como ayuda mental - espiritual no está atada a ningún límite nacional. Para el médico francés Maximilien Isidore Simon, según explicó en su libro Déontologie médicale del año 1845 , sólo se puede permitir o prohibir aliviar la muerte del enfermo con medios físicos y mentales, pero no se puede acabar con su vida 
activamente. La medicina sería una "rama de la caridad" (branche de la charité"), la "eutanasia real" está en la creencia, en la unión con Dios: "une union intime avec Dieu; là est la véritable euthanasie"(13).

\section{Siglo XX}

Distintos enfoques, que apuntan hacia una nueva orientación, aparecen en los siglos XIX y XX en los médicos y pacientes, en el público y la cultura. La persona que quiera hoy tener una influencia sobre estos enfoques, debe considerar, de igual forma, supuestos materiales e ideales.

De gran significado son la eugenesia y el darwinismo social en el 1900, que lleva a los escritos programáticos de Alfred Ploetz (Die Tüchtigkeit unserer Rasse und der Schutz der Schwachen, 1985), Alexander Tille (Volksdienst. Von einem Socialaristokraten, 1893), Wilhelm Schallmayer (Vererbung und Auslese im Lebenslauf der Völker, 1903), Adolf Jost (Das Recht auf den Tod. Sociale Studie, Göttingen, 1895), Elisabeth Rupp (Das Recht aufden Tod, Stuttgart, 1913) así como también de Karl Binding y Alfred Hoche (Von der Freigabe zur Vernichtung lebensunwerten Lebens, 1920, 1922). El darwinismo social, la eugenesia y el utilitarismo entran en una relación fatal, pero, incluso aquí, deben hacerse las diferenciaciones correspondientes. Impulsos esenciales provienen de Inglaterra. En Alemania, la eutanasia se relaciona con el término activo de la vida por un proyecto de ley constituido por ocho artículos, donde se presenta el caso del paciente tuberculoso Roland Gerkan. "El que tenga una enfermedad incurable, tiene el derecho a recibir ayuda para morir"(14).

En numerosos países se fundaron, a comienzos del siglo XX, sociedades para la eutanasia $y$, en diferentes ocasiones, se promulgaron informes para una legalización de la eutanasia activa. En las correspondientes discusiones tomaron parte médicos, abogados, filósofos y teólogos.

Ya en 1873 aparece en la revista 'Popular Science Monthly', el artículo Euthanasia del ensayista Samuel D. Williams con el informe para eutanasia activa y voluntaria para hombres enfermos sin esperanza: "en todos los casos de enfermedad sin cura y dolorosa, se debería reconocer la tarea del médico tratante, si así lo hubiera manifestado el paciente, de administrar cloroformo u otro anestésico que sobrepasara al cloroformo, de manera de destruir la conciencia de una vez y llevar al enfermo a una muerte rápida y sin dolor"(15). Este estudio puede ponerse al lado del trabajo The new cure for incurables del año 1873 de Lionel A. Tollemache, que se refiere a Moro, creado por el darwinismo utilitario: "la ciencia moderna nos dice que en un mundo sobrepoblado se da una dura lucha por la existencia, de manera que al hombre enfermo, infeliz e inútil, de alguna manera se le empuja, por lo menos, a no disfrutar o a no ser alguien que, probablemente, sería más feliz, más saludable y más útil"(16).

La escasez económica y la amenaza en tiempos de guerra fortalecen los argumentos para la eutanasia como eliminación activa de la vida limitada. El naturalista y monista Ernst Haeckel lo ve según esta perspectiva en su estudio Eternidad, de 1915. Los pensamientos de la guerra mundial sobre la vida y la muerte, religión y teoría de la evolución sustentan la matanza de lisiados y enfermos mentales: "Una pequeña dosis de morfina liberaría, no sólo a estas criaturas dignas de lástima, sino también a sus familiares de la carga fútil y penosa de este ser durante largos años"(17). 
El jurista Binding y el psiquiatra Hoche se atienen al principio de la libre voluntad - en la tradición del Stoa y del utilitarismo - en su informe del año 1920 sobre la eutanasia activa, a la "atención de la voluntad de vivir de todos, incluso de los más enfermos y atormentados y de los que ya no son útiles", lo que lleva a la siguiente conclusión: por cierto, no puede decirse que esté permitido dar muerte al débil mental que se siente feliz con su vida“(18).

Este cambio en el pensamiento de los médicos, juristas y también de los científicos y los hombres de letras, posee su correspondencia o preparación en los trabajos del arte o la literatura -en latinos e historias de realismo y, sobre todo, de naturalismo, donde la eutanasia y la eugenesia son sustentadas o presentadas en su ambivalencia. Theodor Storm, en la historia Ein Bekenntnis (1887), deja al médico Franz Jebe dar muerte a su suplicante esposa Elsi, para liberarla de sus dolores insoportables y sin salida producidos por el cáncer; luego de la muerte de su mujer, este médico se entera por algún medio de que, tal vez, ella podría haber sido salvada y que él no fue advertido por la lectura y la literatura científica. Ahora, lo absorbe la "santidad de la vida", que coloca límites infranqueables: "frente al misterio, ningún hombre, ningún hombre de ciencia debe tender su mano, cuando sólo lo hace al servicio de la muerte pues se transforma en un asesino desalmado".

En la población también se sustentan conceptos parecidos. En los años veinte, antes del Tercer Reich, el psiquiatra infantil Ewald Meltzer envió un cuestionario en la perspectiva de una reglamentación, posteriormente legal que, según su parecer, llevaba en la práctica una prohibición. La pregunta: “¿aprobaría usted, bajo cualquier circunstancia, el acortamiento sin dolor de la vida de su hijo, después que un experto determinara que su hijo tiene una enfermedad incurable?" se respondió, según esta encuesta y dejando consternado a Meltzer, de la siguiente manera: el $73 \%$ de los padres respondió que sí, mientras que el 27\% respondió que no. "Esto no me lo habría esperado. Lo contrario me hubiese parecido más verosímil»(19).

Bajo el Nacionalsocialismo, la eutanasia recibe una connotación negativa: se asesinaba a los enfermos y personas que sufrían. En este proceso tomaban parte tanto médicos como enfermeras. En los años 1940 y 1941 se practicó oficialmente la eutanasia activa en el Reich alemán, sin fundamentación legal o sólo bajo referencia a un escrito privado de Hitler de fines de octubre y en referencia a la orden antedatada 1.9.1939: "El Jefe del Reich y el Dr. Brandt son responsables de ampliar las autorizaciones a determinados médicos para que, según el parecer humano, decidan que pueden ser catalogados como candidatos a la eutanasia los enfermos incurables mediante el dictamen crítico del estado de su enfermedad". Bajo la presión de la iglesia católica y de la iglesia evangélica, se llega, el 24 de agosto de 1941, a una interrupción oficial de las acciones de eliminación, las cuales llevaron a más de 70000 de personas a ser víctimas de estos actos. Estos asesinatos se practicaron de manera no oficial("eutanasia ilícita") hasta el término de la guerra $(20)$.

En el Juicio de Nuremberg (1946 1947) se juzgó esta forma ilegal e inmoral de la eutanasia activa sin aclaración y consentimiento o en contra de la voluntad de los afectados. Según estas experiencias, con diferentes juramentos y declaraciones se busca evitar la repetición de una Medicina sin Crueldad (título de un libro de Alexander Mitscherlich y Fred Mielke del año 1946), especialmente para las distintas disciplinas médicas. 


\section{Presente}

En nuestros tiempos se sustentan diferentes opiniones sobre la eutanasia; también es variada la realidad en los distintos países del mundo. Reiteradamente se llega a iniciativas que apuntan a la legalización de la eutanasia. Una amplia atención ha encontrado la reglamentación legal de Holanda. Estudios médicos, psicológicos y sociológicos han llevado, en la actualidad, a nuevos conocimientos sobre el trato con la muerte y el fallecimiento.

Según la ley holandesa, el término de la vida por medio del médico se considera como un delito; sin embargo, puede ser visto bajo la suposición de determinadas condiciones de cuidado, y conforme al principio del consentimiento de un procesamiento penal: libre voluntad del enfermo informado por medio del médico tratante para el paciente con dolor inaguantable, estado irreversiblemente incurable, consulta a un segundo médico no incluido en la terapia, realizado por el médico tratante con el mayor cuidado posible, informe escrito, notificación al médico forense y al abogado. Según una encuesta empírica, en los Países Bajos en 1995, de 136000 moribundas, a 3200 se les practicó la eutanasia activa, la que en su mayoría es aprobada por los médicos holandeses; 9700 pacientes habían solicitado este tipo de muerte; 900 fueron asesinados por los médicos sin formular una petición expresa, puesto que consideraban innecesario otro tratamiento; en un $41 \%$ los médicos fueron obligados a presentarse (21).

Los movimientos como, por ejemplo, los hospicios, la medicina paliativa y los grupos de autoayuda, abogan por la humanización en el trato con los moribundos y pretenden contribuir, de esta manera, a superar la separación de la medicina y la vida y, al mismo tiempo, contrarrestar la difusión de la eutanasia $\operatorname{activa}(14,22)$.
Las interpretaciones de la eutanasia son controversiales en la filosofía y teología actuales, en el juicio ético de la eutanasia activa y en la pregunta sobre la diferencia entre eutanasia activa y pasiva como eutanasia directa e indirecta(23).

La actitud hacia la eutanasia muestra, finalmente, notables desviaciones entre los partidarios de las diferentes religiones; la exigencia de una conservación incondicional de la vida disminuye, según una investigación empírica(24) de médicos católicos a protestantes, pasando por los judíos, así como también cambian las opiniones sobre la eutanasia y sus distintas formas.

En la actualidad, en los medios de comunicación, se pone mayor atención a la muerte y al fallecimiento - con publicaciones, simposios, con las correspondientes relaciones e iniciativas institucionales. Se caracteriza por igual la represión y el silencio; en general, la relación con el morir y la muerte. La minoría de las personas ha experimentado directamente la agonía y la muerte, se ha preparado, o conoce formas de comportamiento; en muchas predomina un sentimiento de desorientación y sin sentido respecto de la muerte.

Al mismo tiempo, las posibilidades de la medicina han crecido bajo una suerte de presión por alargar la vida y aplazar la muerte, así como también por predecir su llegada. El progreso médico no puede suprimir las condiciones fundamentales de la vida humana: tener un término y sobre este término también estar al tanto. La medicina debe conocer sustancialmente esta paradoja o dilema fundamental: querer sanar lo que, después de todo, no se puede sanar. El médico experimenta con dolor, en el trato con los moribundos, los límites de su propio conocimiento y capacidad.

La muerte se refiere a diferentes ámbitos: desintegración física, aislamiento 
social, tristeza de vivir y miedo a la muerte. Estos ámbitos, de ninguna manera, se hacen realidad en el paralelismo o en la armonía. La muerte social puede ocurrir antes de la muerte física, la muerte espiritual, antes de la muerte del alma. La muerte natural se opone a la muerte violenta. El miedo a morir no es idéntico al miedo a la muerte, los dolores pueden referirse tanto al cuerpo como al alma.

Se pueden diferenciar más fases o dimensiones en el transcurso de la muerte, gran resonancia encontró en la medicina el desglose de Elisabeth Kübler - Ross: negación, obstinación, debate, resignación, aceptación(25). Estas fases pueden no ser experimentadas en este orden; pueden extenderse, así como también aumentar o cambiar. Junto a las fases y dimensiones de la muerte existen diferentes etapas de la experiencia: la conciencia puede existir en su claridad, además, es posible que se presenten reducciones temporales o fundamentales como decaimiento corporal y espiritual.

Todas las particularidades y desviaciones en los estadios y etapas de la muerte pueden ser prevenidas con empatía y humanidad. Siempre hay formas de apoyo y consuelo; en las etapas inferiores sólo está la asistencia, el contacto corporal, el sonido de la voz, el movimiento de los ojos; en las etapas superiores está la unión del habla y del espíritu. En especial significado para la compañía del moribundo, para su supervivencia en el recuerdo y la preparación para su propia muerte, tienen el contexto social y el mundo de la cultura.

Sin embargo, los límites de la participación y de la comunicación no deben ser pasados por alto; en las profundidades de la existencia y la muerte tener la posibilidad de poder hablar, oportunidad que no todos tienen. Pero también el silencio y la soledad poseen un valor. Acertada es la frase del filósofo religioso católico Romano Guardini:
"La vida del hombre se realiza entre el silencio y el habla, la tranquilidad y la palabra"(26).

La muerte y el morir significan antelación, suceso y recuerdo. La muerte es anticipada por el afectado y, en la mayoría de los casos, no se experimenta en un cumplimiento inmediato; la muerte es un suceso para los otros o para el mundo circundante. La eutanasia no es sólo una problema de la autonomía individual, sino también está relacionada con la reacción de la familia y la sociedad ante la enfermedad, limitación y edad; la muerte y el morir tienen su lado económico, que se modela por la relación entre las generaciones. Los intentos de suicidio señalizan conflictos individuales, pero también culturales y sociales y, por lo general, corresponden a llamadas de atención a los parientes y amigos.

El hombre vive y muere en la sociedad; la sociedad está inserta en el mundo de la cultura. El moribundo recibe apoyo y sosiego no sólo de su familia, amigos, colegas de trabajo, la tranquilidad puede venir también de valores individuales de la cultura - la literatura, la pintura, la música, la filosofía y la teología. Junto a la trascendencia del pensamiento se encuentra la inmanente trascendencia de la cultura, de la que todo hombre forma parte, que está llena de ayuda y de orientación para el cuerpo, el alma y el espíritu.

Decidida está la orientación del hombre hacia su propia muerte, decidido está el comienzo de la muerte en la realidad social, decidida está la inmanente unión de la vida y la muerte y de la muerte y la cultura. De Montaigne viene la frase: "Tú no mueres porque estés enfermo, mueres porque vives(27)." Un informe para el cambio práctico preciso contiene la convicción fundamental del escritor existencialista Albert Camus; "La única solidaridad real entre los hombres es la solidaridad ante la muerte." 


\section{Perspectivas}

Las raíces históricas de la eutanasia, en espectro, entre el acortamiento de la vida y la ayuda a morir se remontan a la Antigüedad están dadas en todas las diferencias que, sin duda, están presentes entre las posiciones ideales y los cambios en la realidad.

La comprensión y la valoración de la eutanasia, en la actualidad, son tan uniformes como en el pasado. La eutanasia no se puede comparar, bajo ninguna circunstancia con la eutanasia activa, ni menos con el asesinato sin consentimiento o contra la voluntad. Centrales son las siguientes diferencias o tipos de eutanasia:

\section{Eutanasia exterior y eutanasia interior;}

2. Eutanasia activa y eutanasia pasiva;

3. Eutanasia directa y eutanasia indirecta;

4. Eutanasia heterónima y eutanasia autónoma;

5. Causa de la eutanasia; y

\section{Sujeto ejecutor de la eutanasia}

En la realidad, ya no se respetan los límites entre estos diferentes tipos, pero no necesariamente habla en contra de las diferencias conceptuales. Junto a los pasos y las uniones hay incluso más diferencias. Sobre bases teóricas, se puede hacer diferenciaciones de fondo de determinados tipos de eutanasia; quien no vea la diferencia ontológica ni teológica, encontrará aún menos significativa la diferencia entre eutanasia activa y eutanasia pasiva.

La eutanasia activa contra la voluntad del afectado no puede ser justificada bajo ningún punto de vista; el juicio sobre la eutanasia activa heterónima debe ser categóricamente negativo. Hay que atenerse, en principio, al consentimiento de la eutanasia pasiva; incluso así podemos llegar a la carencia de humanidad sobre el tema. Detrás de la eutanasia existe un pensamiento eugenésico, por lo tanto, nadie puede pasar por alto las disposiciones que se presentan en una dictadura, como el caso del Tercer Reich. Existe, en la actualidad, sin duda alguna el temor de una eugenesia individual, libre y democráticamente legitimada.

Quien quiera evitar repeticiones negativas de la historia, no debe dirigir sus ideas y emociones al Tercer Reich. El absolutismo de los países en contra de la cultura, filosofía, religión y ciencias trae consigo el peligro inmanente. El ser humano siempre ha tenido objetivos políticos y sociales que pueden poner en peligro a los enfermos y a los adultos mayores. Las Ciencias Naturales y la Medicina no pueden tener libertad plena para la práctica de sus investigaciones.

Sobre el deseo del paciente de morir y la eutanasia activa autónoma practicada por el médico se dividen las opiniones. Cada vez más gente opta por esta posibilidad. El pensamiento en contra de la legalización de la eutanasia activa pesa, pero poco. Los resultados de la relación médico - paciente son peligrosos: los enfermos y los moribundos pueden perder su confianza en la medicina y en la conservación de la vida médicamente hablando; ya el hombre considera la eutanasia activa como una posibilidad que no lo hará pasar por el doloroso proceso de morir.

La autonomía del paciente se enfrenta con la autonomía del médico. Bajo ningún motivo se puede permitir el acto de asesinato por parte del médico, aunque éste sea el deseo 
de su paciente. Debemos convivir con la pluralidad de orientaciones éticas existentes y la gran cantidad de religiones. Debemos buscar un costo ético mínimo y pedir protección legal en caso de que ésta no exista, debemos dar a conocer el valor de la vida a los pacientes en estado terminal, debemos proporcionar una orientación ética positiva al médico tratante o al personal del hospital donde se cuidan enfermos sin mayores esperanzas.
Gran parte de las responsabilidades éticas principales sobre esta materia está en manos de la medicina, la sociedad y los países. No se debe pensar que la eutanasia es una forma de morir rápida y sin dolor. Tampoco hay que pedirle al médico el acortamiento de la vida, ya que la ética es igual para todos. El dolor nunca ha justificado ni justificará la ayuda a morir, son otras las cuestiones que debemos analizar para llegar a un planteamiento sano al respecto.

\section{Referencias}

1. Singer P. Praktische Ethik, a.d.Engl. (1980). Stuttgart; 1984: 169.

2. Gruman GJ. An historical introduction to ideas about voluntary euthanasia: with a bibliographic survey and guide for interdisciplinary studies. In: Omega 4 1973; (2)87-138

3. Triche ChW, Triche DS. The euthanasia controversy 1812-1974. New York; 1975.

4. Vanderpool HY. Death and dying: Euthanasia and sustaining life. I. Historical aspects. In: Warren T. Reich. Encyclopedia of bioethics. Bd. 1, New York 2 1995: 554-563

5. Ariès Ph. Geschichte des Todes, a.d.Franz. (1978). München; 1982.

6. Imhof AE. Die Lebenszeit. Vom aufgeschobenen Tod und der Kunst des Leben. München; 1988.

7. Sueton: Caesarenleben, a.d.Lat. (um 120 n.Chr.). Stuttgart; 1986: 157.

8. Lichtenthaeler Ch. Der Eid des Hippokrates. Köln; 1984.

9. Bacon F. De dignitate et augmentis scientiarum. a.d. Lat. (1623). Stuttgart: Bad-Cannstatt; 1963: 594f.

10. Morus Th. Utopia. a.d. Lat. (1516). Hamburg: Reinbek; 1983: 81

11. Andreae V. Christianopolis. a.d. Lat. (1619). Stuttgart; 1975: 137.

12. Hufeland ChW. Enchiridion medicum oder Anleitung zur medicinischen Praxis. Berlin; 1836: $734 f$. 
La Eutanasia: pasado y presente - D. von Engelhardt

13. Simon M I. Déontologie médicale. Paris; 1845.

14. Mitgeteilt von Wilhelm Ostwald. In: Das monistische Jahrhundert 1913; 2: 169-173.

15. Williams SD. Euthanasia. In: Popular Science Monthly 1873 (may); 91.

16. Tollemache LA. The new cure of incurables. In: Fortnightly Review 1873 (febr.): 218-230.

17. Haeckel E. Ewigkeit. Weltkriegsgedanken über Leben und Tod, Religion und Entwicklungslehre. Berlin; 1915.

18. Binding K, Hoche A. Von der Freigabe zur Vernichtung lebensunwerten Lebens. 1920: S. $28 \mathrm{f}$.

19. Meltzer E. Das Problem der Abkürzung “lebensunwerten” Lebens: Halle; 1925: 88.

20. Mitscherlich A, Mielke F. Medizin ohne Menschlichkeit. Dokumente des Nürnberger Prozesses, Heidelberg 1947 (unter dem Titel: Das Diktat der Menschenverachtung). Frankfurt a.M; 1978.

21. Gordijn B. Euthanasie in den Niederlanden - eine kritische Betrachtung (= Berliner Medizinethische Schriften, H. 19). Dortmund; 1997: 17-27

22. Saunders C, Hg. Hospiz und Begleitung im Schmerz, a.d. Engl. 1990. Freiburg i.Br; 1993.

23. Birnbacher D. Ethische Aspekte der aktiven und passiven Sterbehilfe. In: Hepp H, Hg. Hilfe zum Sterben? Hilfe beim Sterben. Düsseldorf; 1992: 50-73.

24. Crane D. The sanctity of social life: Physicians 'treatment of critically ill patients. New York; 1979.

25. Kübler-Ross E. Interviews mit Sterbenden. a.d. Engl. 1969. Stuttgart; 1969: 91982.

26. Guardini R. Tugenden. Meditationen über Gestalten sittlichen Lebens. Würzburg; 1963: 192.

27. De Montaigne M. Essais. a.d. Franz. (1580/95). Zürich; 1953: 868. 